J. Clin. Chem. Clin. Biochem.

Vol. 14, 1976, pp. 569-576

\title{
Isolierung von Komponenten menschlicher Magenschleimhaut-Pepsinogene und -Cathepsine mit präparativer Polyacrylamidgelelektrophorese zur Herstellung spezifischer Immunseren
}

\author{
Von W. Rapp und H. E. Lehmann ${ }^{1}$ ) \\ Mediz inische Universitätsklinik Heidelberg und Institut für Nuklearmedizin am Deutschen Krebsforschungsz entrum \\ Heidelberg
}

(Eingegangen am 18. November 1975/9. Juni 1976)

Zusammenfassung: Mit der präparativen Polyacrylamidgelelektrophorese wurden aus menschlichen Magenschleimhautextrakten Komponenten der immunchemisch verschiedenen Pepsinogengruppen I, II und des Magenschleimhautcathepsins gewonnen. Die Immunisierung von Schafen und Kaninchen ergab spezifische Immunseren, die gegen die drei verschiedenen Systeme der Magenschleimhautproteasen gerichtet waren.

\section{Isolation of components of pepsinogens I, II and mucosal cathepsin of luman gastric mucosa by preparative poly- acrylamide gel electrophoresis, and the preparation of specific antisera}

Summary: Components of pepsinogens I, II and mucosal cathepsin from human gastric mucosa were purified from gastric extracts by preparative polyacrylamide gel electrophoresis. Specific antisera directed against three different systems of gastric proteases were raised by immunization of sheep and rabbits.

\section{Einführung}

Mit der von Uriel (1) konzipierten Agargelenzymelektrophorese (Enzymelektrophorese) haben wir vier elektrophoretisch unterscheidbare Magenschleimhautproteasen identifiziert (2). Aufgrund ihrer immunogenen und antigenen Eigenschaften konnten wir diese Proteasen in drei immunchemisch unterscheidbare Gruppen einordnen (3). Die beiden ersten Gruppen mit der größten elektrophoretischen Mobilität erwiesen sich als Pepsinogene (4). Die dritte, langsam wandernde Proteasengruppe unterschied sich von den Pepsinogenen durch ingre besondere Stabilität im neutralen und alkalischen Milieu nach vorhergehender Säureaktivierung (4). Von Hirsch-Marie wurde in zahlreichen Untersuchungen innerhalb des gleichen methodischen Bezugsystems bewiesen, daß es sich bei diesen Pepsinogenen um Proenzyme der im Magensaft nachweisbaren Pepsine handelt $(5,6)$.

Durch methodische Verbesserungen der Enzymelektrophorese gelang Samloff (7) die weitere Differenzierung und Charakterisierung der Magenschleimhautproteasen (8). Die von Samloff vorgeschlagene Klassifikation der Magenschleimhautproteasen unterscheidet zwei immunologisch differente Pepsinogengruppen und eine in der Enzymelektrophorese langsam wandernde Protease (,,slow migrating protease"), die sich von den Pepsinogenen durch ihre Alkalistabilität unterscheidet (8). Die Pepsinogengruppe I zeigt die größte elektrophoretische Mobilität und besteht aus deutlich unterscheidbaren Enzymbanden 1-5. Die Pepsinogengruppe II wandert langsamer als die Pepsinogengruppe I und besteht aus den Enzymbanden 6 und 7.

Whitecross et al. (9) und Mangla et al. (10) konnten durch weitere methodische Modifikationen der Enzymelektrophorese bis zu elf verschiedene Banden mit proteolytischer Aktivität beschreiben.

Die Isolierung von immunchemisch homogenen Pepsinogengruppen I bzw. II wurde bisher noch nicht berichtet. In der vorliegenden Arbeit wurde versucht, mit der präparativen Polyacrylamidgelelektrophorese einzelne Komponenten der beiden Pepsinogengruppen sowie der langsam wandernden Proteasen aus menschlichen Magenschleimhautextrakten zu isolieren. Gegen diese isolierten Proteasen wurden spezifische Antiseren hergestellt. Damit waren die Voraussetzungen für die Reinigung von menschlichen Pepsinogenen und Magenschleimhautproteasen nach immunchemischen Gesichtspunkten durch Immunadsorptionstechniken geschaffen (11).

1) Mit Unterstützung der Deutschen Forschungsgemeinschaft. 


\section{Material und Methoden}

\section{Material}

Chirurgische Resektionspräparate wurden nach Entnahme von Proben zur histologischen Beurteilung tiefgefroren und bis zur wciteren Verarbeitung und Extraktion bei $-20^{\circ} \mathrm{C}$ aufbewahrt. Die Extraktion erfolgte mit physiologischem Puffer $(10 \mathrm{mmol} / 1$ Natriumphosphat $\mathrm{pH} 7,2 ; 150 \mathrm{mmol} / 1$ Natriumchlorid). Das Verhältnis von Gewebe zu physiologischem Puffer betrug 1:3 (vol/vol). Das mit dem Ultraturray (Type TP 18-10, JANKE und KUNKEL KG, Staufen i. Br., Deutschland) homogenisierte Gewebe wurde bei $75000 \mathrm{~g} 30$ min zentrifugiert. Der zur Isolierung der Proteasen verwendete Überstand hatte eine Proteinkonzentration von $10-20 \mathrm{~g} / \mathrm{l}$.

\section{Immunologische Geldiffusionsmethoden}

Die immunelektrophoretischen Analysen und die zweidimensionale Geldiffusion nach Ouchterlony wurden in Agarose $(10 \mathrm{~g} / \mathrm{l}, \mathrm{SERVA}$, Heidelberg) und in Natrium-Veronalpuffer $(0,05 \mathrm{~mol} / 1, \mathrm{pH} 8,2)$ in dem UGI-System (DESAGA, Heidelberg) nach früheren Angaben (12) durchgeführt. Zum Nachweis proteolytischer Aktivitäten in den Immunpräzipitaten wurde die ursprünglich beschriebene Methode (3) wie folgt abgeändert: Die gewaschenen Gelplatten wurden zunächst während ciner Stunde in neutraler Lösung von Hämoglobin vom Rind $(6 \mathrm{~g} / \mathrm{l}$ dest. Wasser) getränkt. Nach Abgießen der Lösung wurde die Gelplatte während $20 \mathrm{~min}$ in einer Lösung von $6 \mathrm{~g} / \mathrm{l}$ Hämoglobin in $0,1 \mathrm{~mol} / 1 \mathrm{HCl}$ bci pH 1,2 aktiviert und inkubiert. Anschließend wurden die Platten in Trichloressigsäure fixiert, unter Filterpapier getrocknet und mit Amidoschwarz angefärbt. Proteolytische Aktivitäten stellten sich als transparente Aussparungen auf blauem Untergrund dar.

\section{Radiale Geldiffusion}

Sie erfolgte nach den Angaben von Mancini et al. (13) in AgaroseVeronalpuffer ( $10 \mathrm{~g} / \mathrm{l}$ Agarose). Das Verhältnis der Agarose zu den Antiseren betrug 1:9 bzw. 1:19. Es wurden jeweils Probenmengen von $5 \mu$ aufgetragen. Die Auswertung erfolgte nach Abschluß der Diffusion. Zur Erstcllung von Bezugspunkten wurden arbiträre Standardproben von Magenextrakten bzw. des jeweiligen Ausgangsmaterials verwendet.

\section{Agarose-Enzym-Elektrophorese}

Die Durchführung erfolgte im Prinzip nach Samloff (8) unter Einbeziehung von mehreren Modifikationen. Agarose $(20 \mathrm{~g} / \mathrm{l})$ wurde auf Glasplatten von $8 \times 16,5 \mathrm{~cm}$ und $16,5 \times 16,5 \mathrm{~cm}$ mit Hilfe eines $1 \mathrm{~mm}$ starken Kunststoffrahmens und einer aufgelegten zweiten Glasplatte in einer $1 \mathrm{~mm}$ dicken Schicht aufgegossen. Nach Verfestigung des Gels wurde die obere Glasplatte vorsichtig abgezogen. Am kathodischen Ende wurden Auftrennreservoire von $0,1 \times 1,0 \mathrm{~cm}$ ausgestanzt. Als Gelpuffer wurde Tris-Glycin-Puffer $(0,1 \mathrm{~mol} / \mathrm{l} ; \mathrm{pH} 8,3)$ und als Trennpuffer Tris-Glycin-Puffer $(0,065 \mathrm{~mol} / \mathrm{l} ; \mathrm{pH} 8,2)$ verwendet. Die Verbindung zu den Puffertrögen wurde mit Filterkarton hergestellt. Die Auftrennung erfolgte bei 800 Volt ( $20 \mathrm{~mA} /$ Platte) während 15-20 Minuten. Die Kühlung erfolgte durch fließendes Leitungswasser. Die elektrophoretische Auftrennung wurde durch ein Farbstoffgemisch von Bromphenolblau-Albumin und Cyanocobalamin kontrolliert (14). Die Proben wurden direkt in die Auftrennungsreservoire nach vorhergehender Dialyse gegen den Trennpuffer eingefüllt. Bei optimaler Auftrennung betrugen die Entfernungen der Farbsubstanzen von der Auftragsstelle anodenwärts: Albumin-Bromphenolblau $=6,2 \mathrm{~cm}$, nicht gebundenes Bromphenolblau $=11 \mathrm{~cm}$. Nach der Auftrennung wurde die Gelplatte in Hämoglobin (vom Rind, $6 \mathrm{~g} / \mathrm{l}$ ) mit $0,1 \mathrm{~mol} / \mathrm{l} \mathrm{HCl}$ auf pH 1,2 eingestellt, während 10 Minuten inkubiert und nach $\mathrm{Ab}$ guß des Substrates in einer feuchten Kammer bei Raumtemperaturr während 10 Minuten nachinkubiert. Anschließend wurde die Platte in Trichloressigsäure $(50 \mathrm{~g} / \mathrm{l})$ fixiert, unter Filterpapier getrocknet und mit Amidoschwarz gefärbt.

\section{Präparative Polyacrylamid-Gel-Elektrophorese}

Es wurde das Verfahren nach Allen (15) mit diskontinuierlichen Voltgradienten verwendet. Als Trenneinheit wurde die UGI und eine von uns modifizierte Trennkammer zur horizontalen Auf- trennung benützt. Die Kammer bestand aus Polyvinylchlorid, der Boden und die Deckplatten waren aus Hartglas gefertigt. Die Dimensionen für das Gel betrugen $9 \times 12 \mathrm{~cm}$ mit auswechselbarem Rahmen aus Polyvinylchlorid für Geldicken von $0,5 \mathrm{~cm}$. Die Trennstrecke betrug insgesamt $12 \mathrm{~cm}$. Das Gel wurde über vartikale Verbindungströge bis zu den Pufferbehältern durchgegossen. Die Präparation des Gels erfolgte im Prinzip nach Allen (16). In der anodischen Pufferbrücke und in dem 7,5 cm langen Trennbereich wurde $15 \%$ Gel verwendet. Die Länge des Sammelgels $(3,5 \%)$ betrug $1,0 \mathrm{~cm}$. In der anodischen Brücke befand sich $5 \%$ Gel. Zur Herstellung des Gels wurde ein Tris-Puffer von pH 9,0 mit 4,5 mmol/l im Sammelgel und $30 \mathrm{mmol} / 1$ im Trenngel verwendet. Als Elektrodenpuffer und für den kathodischen Brückengel benützten wir Tris-Boratpuffer $(62 \mathrm{mmol} / \mathrm{l} ; \mathrm{pH} 9,0)$. Die Probe $(2-4 \mathrm{ml})$ mit einem Proteingehalt von $10-80 \mathrm{mg}$ wurde unter dem Brückengel durch Unterschichtung aufgebracht. Seitlich wurde zur fortlaufenden Kontrolle im Gel Bromphenolblau markiertes Albumin eingefüllt. Die Spannung wu rde während der ersten 15 Minuten von 80 Volt kontinuierlich auf 400 Volt Trennspannung gesteigert. Die weitere Auftrennung erfolgte während 60 Minuten bei 400 Volt unter fortlaufender Kühlung durch Leitungswasser. Nach optimaler Auftrennung betrug die Entfernung des freien Farbstoffes anodenwärts 7,5 cm. Ein seitlicher Streifen von $1,5 \mathrm{~cm}$ Breite wurde zur Kontrolle mit Trichloressigsäure $(100 \mathrm{~g} / \mathrm{l})$ fixiert und mit Coomassie $R 250$ (SERVA, Heidelberg) angefärbt. Das Gel wurde auf einen speziell angefertigten, in 25 Segmenten unterteilten Schneidetisch aufgelegt und mit einem Stahldraht in 25 Streifen zu je $3 \mathrm{~mm}$ Trennlänge geschnitten. Die Gelstreifen wurden ziweimal bei $-20^{\circ} \mathrm{C}$ gefroren, aufgetaut und anschließend mit einem Spatel homogenisiert. Elution, Dialyse und Konzentration des Eluates erfolgten über Glasfritten mit einem Unterdruck-Konzentriertopf (DESAGA, Heidelberg) mit Hilfe von Visking-Dialysierschläuchen innerhalb eines Arbeitsganges. Die einzelnen Gelstreifen wurden jeweils mit $100 \mathrm{ml}$ physiologischem Puffer (s. ,Material") während 35 Stunden eluiert und das Eluat auf 2-4 ml konzentriert. Zur weiteren Untersuchung wurden die Eluate der einzelnen Gelstreifen einer Auftrennung auf gleiche Volumen eingestellt.

\section{Zweidimensionale Immunelektrophorese}

Die Durch führung erfolgte nach Laurell (17). Magenextrakt $(10 \mathrm{~g} / \mathrm{l})$ wurde analog zur Enzymgel-Elektrophorese in Dünnschichtgel aufgetrennt. Nach der elektrophoretischen Auftrennung wurde ein Gelstreifen entfernt und an Agarosegel $(20 \mathrm{~g} / \mathrm{l})$ von gleicher Länge und $5 \mathrm{~cm}$ Breite angelegt. Diese Gelschicht enthielt 9 Teile Agarose und 1 Teil Antiserum, das gegen die Pepsinogengruppe I bzw. II gerichtet war. Die elektrophoretische Auftrennung in der zweiten Dimension erfolgte bei 60 Volt während 4 Stunden. Eine simultane Auftrennung des gleichen Magenextraktes diente der Darstellung der proteolytischen Aktivitäten in der Enzymelektrophorese.

\section{Proteinbestimmung}

Es wurde die modifizierte Folin-Methode nach Lowry et al verwendet (18).

\section{Enzymatische Bestimmung der proteolytischèn Aktivität}

Es wurde die modifizierte Methode nach Anson varwendet (19). Die Pepsinaktivitäten wurden in Internationalen Einheiten (IU) angegeben. Definitionsgemäß ist eine IU die $1 \mu \mathrm{mol}$ Tyrosin äquivalente Menge an säurelöslichen Produkten der enzymatischen Hydrolyse.

\section{Analytische Polyacrylamid-Gel-Elektrophorese}

Es wurde das Verfahren nach Allen (15) mit diskontinuierlichem Spannungsgradienten verwendet. Diskontinuierliche Gelgradienten wurden mit dem Gelsystem 2 (16) von $3 \%$ $(1,0 \mathrm{~cm}), 6 \%(0,5 \mathrm{~cm}), 8 \%(1,0 \mathrm{~cm}), 12 \%(5,0 \mathrm{~cm})$ gegossen. Fixierung und Entfärbung erfolgten mit Trichloressigsäure und Coomassie R 250.

\section{Herstellung von Immunseren}

Isolierte Proteasefraktionen ivurden mit kompletten Freund'schen Adjuvans (Difco) emulgiert. Kaninchen wurden nach einem bewährten Schema (12) animmunisiert und in etwa 20tägigen Ab- 
ständen während 3-5 Monaten nachimmunisiert. Pro Kaninchen und Immunisierung wurden $100 \mu \mathrm{g}$ Protein verwendet. Schafe wurden mit dem gleichen Material subcutan animmunisiert und in 4wöchigen Abständen insgesamt 3-6 mal intramuskulär nachimmunisicrt. Pro Immunisierung wurden jetveils $100 \mu \mathrm{g}$ Protein pro Schaf verwendet.

\section{Ausgangsmaterial}

Zur Auftrennung von Pepsinogengruppe I und Pepsinogengruppe II wurden übenwicgend Schleimhau textrakte aus dem Corpusbereich verwendet, die die wichtigsten Komponenten der beiden Pepsinogengruppen I und II enth ielten. Der zur Isolierung der langsam-wandernden Protease erforderliche Magencxtrakt wurde wie folgt von der Auftrennung behandelt: 10 Volumen eines Magenschleimhautextraktes wurden mit 1 Volumen $1,0 \mathrm{~mol} / 1$ $\mathrm{HCl}$ während einer Stunde bei Raumtemperatur inkubiert und anschließend bei $74000 \mathrm{~g} 30$ Minuten zentrifugiert. Das Präzipitat wurde verworfen, der Überstand wurde mit $1,0 \mathrm{~mol} / 1 \mathrm{NaOH}$ auf $\mathrm{pH} 7,0$ cingestellt und anschließend durch Ultrafiltration auf eine Proteinkonzentration von $45 \mathrm{~g} / \mathrm{l}$ gebracht. Sämtliche Magenextrakte wurden vor der Auftrennung gegen den Trennpuffer dialysiert.

\section{Ergebnisse}

\section{Pepsinogengruppe I und II}

Die Auftrennung von Magenextrakten von $40 \mathrm{mg}$ Protein mit der präparativen Polyacrylamid-Gel-Elektrophorese ergab elf Proteinfraktionen mit einem unterschiedlichen Protein- und Proteasengehalt (Tab. 1). Die Untersuchung der einzelnen Fraktionen mit der analytischen Polyacrylamid-Gel-Elektrophorese zeigte Proteinbanden unterschiedlicher Mobilität (Abb. 1). Proteolytische Aktivitäten wurden in vier Fraktionen nachgewiesen (Tab. 1). Diese proteolytischen Aktivitäten zeigten in der Enzymelektrophorese unterschiedliche Verteilungsmuster (Abb. 2a). Insgesamt konnten wir in der Enzymelektrophorese mindestens acht proteolytische Banden unterschiedlicher Mobilität unterscheiden. Die Banden 1-5 mit der größten elektrophoretischen Mobilität gehörten zur Pepsinogengruppe I, während die Banden 6,7 und 8 der Pepsinogengruppe II zugeordnet wurden. Eine deutliche Trennung der beiden Pepsinogengruppen erfolgte lediglich in der anodischen Fraktion Nr. 11 mit der größten elektrophoretischen Mobilität (Abb. 2a). In den drei anderen Fraktionen mit proteolytischer Aktivität beobachteten wir ein Überlappen der beiden Pepsinogen. systeme (Abb. 2a, Tab. 1).

Bei der Verwendung eines geringeren Auftrags von $24 \mathrm{mg}$ Protein wurde ein besseres Trennergebnis erzielt. Hier konnten bei den langsam wandernden Fraktionen die Pepsinogengruppe II von der Pepsinogengruppe I abgetrennt werden (Abb. 2b). Die Ausbeute reiner Pepsinogengruppen betrug pro Auftrennung $0,5 \mathrm{mg}$ Protein.

\section{Langsam wandernde Protease}

Durch die Ansäuerung und Neutralisierung der Magenextrakte konnten wir in der Enzymelektrophorese die Inaktivierung der Pepsinogengruppe I beobachten, während im Bereich der Pepsinogengruppe Il eine Bande noch deutliche proteolytische Aktivitäten aufwies (Abb. 3). In der quantitativen Geldiffusion war in dem angesäuerten Material die Pepsinogengruppe I nicht mehr nachweisbar, während die Pepsinogengruppe II bzw. ihre aktivierten Komponenten eine deutliche Zunahme der immunologisch meßbaren Konzentration im Vergleich zu dem Ausgangsmaterial aufwiesen. Nach der präparativen Polyacrylamid-Gel-Elektrophorese (Auftrag von $22 \mathrm{mg}$ Protein) war die langsam wandernde Protease in vier Fraktionen nachweisbar. In vier weiteren, der Anode zu gelegenen Fraktionen, waren noch schwache proteolytische Aktivitäten und relativ hohe immunologische Aktivitäten der Pepsinogengruppe II vorhanden (Tab. 1). Nach einer erneuten Auftrennung der langsam wandernde Protease enthaltenden Fraktionen (Auftrag von 1,3 mg Protein) konnte eine noch bessere Abtrennung von immunologisch nachweisbaren Komponenten der Pepsinogengruppe II beobachtet werden. Reines Material der langsam wandernden Protease ergab eine Ausbeute von $0,9 \mathrm{mg}$ pro Auftrennung und zeigte in der Enzymelektrophorese 2-3 Banden proteolytischer Aktivität (Abb. 3).

Tab. 1. Präparative Auftrennung von Magenextrakten in der Polyacrylamid-Gel-Elektrophorese. Die einzelnen Gelstreifen wurden eluiert, das Eluat wurde auf gleiches Volumen eingestellt. Die Fraktion 1 (Kathode) hat die langsamste, die Fraktion 16 (Anode) ergibt die größte elektrophoretische Mobilität. In den einzelnen Fraktionen werden der Proteingehalt, die proteolytischen Aktivitäten als auch die immunologisch meßbaren relativen Konzentrationen der Magenschleimhautproteasen verglichen.

Fraktion en

\begin{tabular}{|c|c|c|c|c|c|c|c|c|c|c|c|c|c|c|c|c|}
\hline & & Fra & $\mathrm{ktic}$ & $n c$ & & & & & & & & & & & & \\
\hline & Messung & 1 & 2 & 3 & 4 & 5 & 6 & 7 & 8 & 9 & 11 & 12 & 13 & 14 & 15 & 16 \\
\hline ఏ & Protein (mg/Fraktion) & 2,8 & 0,6 & 1,2 & 2,1 & 3,6 & 0,5 & 0,4 & 0,6 & 1,1 & $1,00,5$ & 0,1 & 0,1 & 0,1 & 0,1 & 0,07 \\
\hline 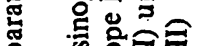 & Aktivität (IU/Fraktion) & & & & & & & & 0,2 & 0,4 & $0,40,3$ & & & & & \\
\hline 운 & $\mathrm{PgI}^{+}$ & & & & & & & & 5,8 & 11,0 & $12,68,9$ & & & & & \\
\hline H>a & $\mathrm{PgII}^{+}$ & & & & & & & & 5,4 & 7,1 & 6,0 & & & & & \\
\hline E. & Protein (mg/Fraktion) & 1,8 & 1,3 & 1,0 & 0,5 & 0,6 & 0,7 & 0,9 & 0,9 & 0,4 & $0,71,5$ & 1,4 & 1,2 & 0,6 & 0,7 & 0,9 \\
\hline 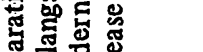 & Aktivität (IU/Fraktion) & & & & & & & 0,7 & 1,6 & 1,1 & $0,8 \quad 0,4$ & 0,2 & 0,2 & 0,1 & & \\
\hline 范茟。 & $\mathrm{PgII}^{+}$ & & & & & & & & & & 6,5 & 7,2 & 9,2 & 7,0 & & \\
\hline & SMP + & & & & & & & 5,1 & 9,0 & 7,0 & 6.0 & & & & & \\
\hline
\end{tabular}

${ }^{+}$relative Konzentration bestimmt mit der radialen Geldiffusion

SMP = Reine Fraktion der langsam wandernden Magenschleimhautprotease. 
$\oplus$

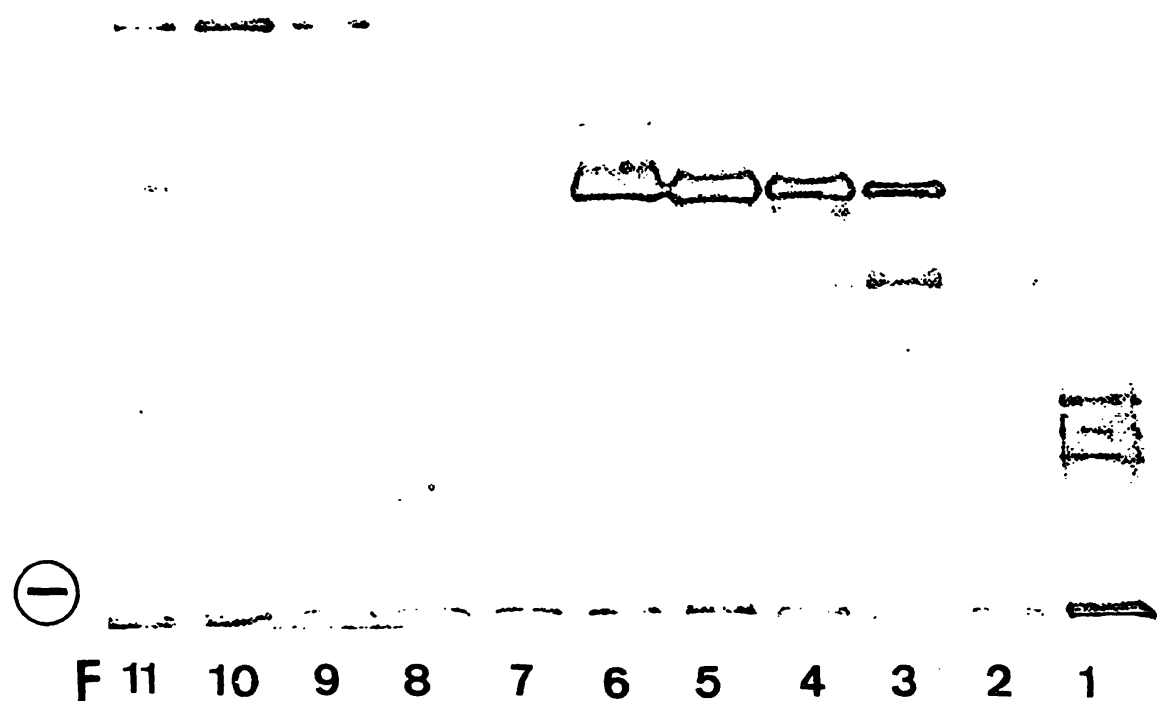

Abb. 1. Elektrophorese in Polyacrylamidgel von isolierten Fraktionen (F) aus Magenschleimhautextrakten. Komponenten der Pepsinogengruppe $I$ sind in $F_{11}$ nachweisbar, Komponenten der Pepsinogengruppe I bzw. II in den übrigen Fraktionen $\left(F_{8-10}\right)$. Vergleiche hierzu Abb. 2 a.

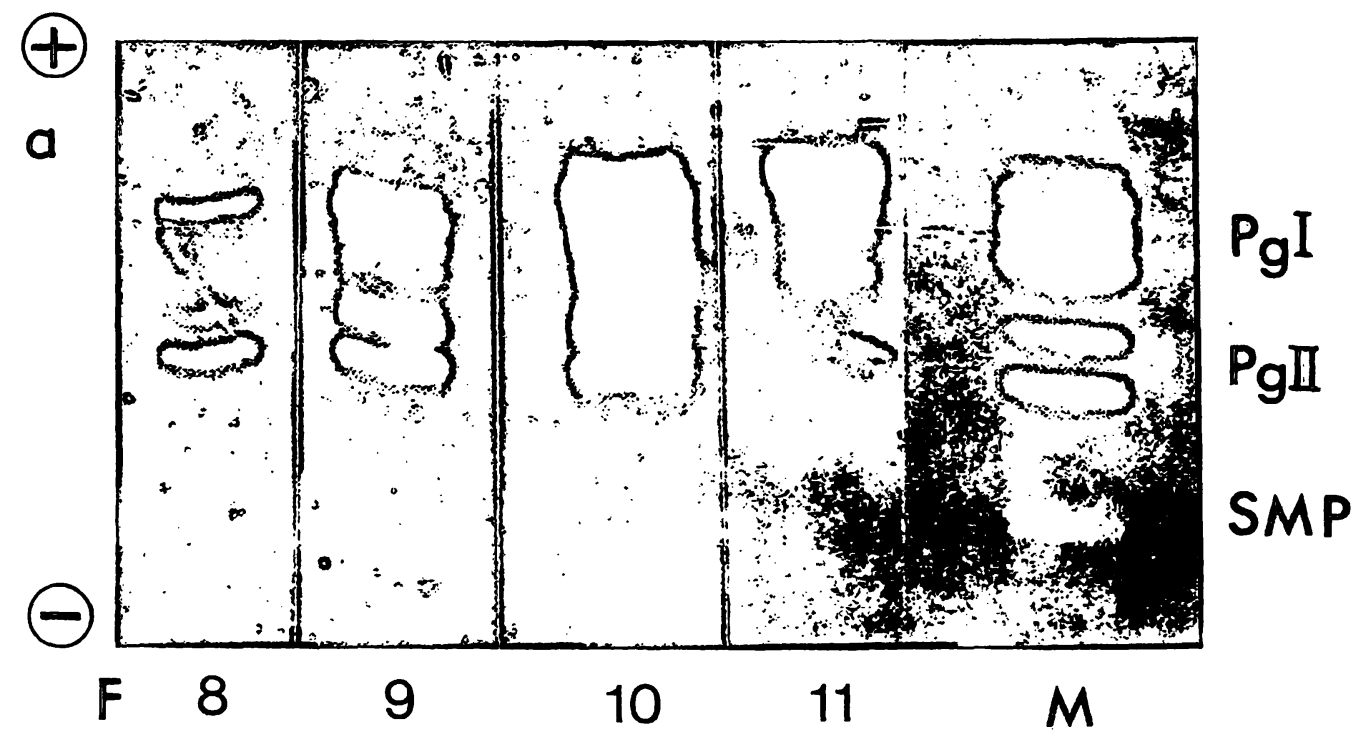

Abb. 2. Agargelenzymelektrophorese isolierter Fraktionen (F) aus Magenschleimhautextrakt.

Abb. 2a. Präparation 1 (Auftrag von $40 \mathrm{mg}$ Protein). $\mathrm{M}$ = Magenextrakt, typisches Verteilungsmuster der proteolytischen Aktivitäten. $\mathrm{F}=$ isolierte Fraktionen. Komponenten der Pepsinogengruppe $\mathrm{I}$ in $\mathrm{F}_{11}$, Komponenten der Pepsinogengruppe I und II in $F_{8,9,10} . \mathrm{SMP}=$ Reine Fraktion der langsam wandernden Magenschleimhautprotease. Pg I = Pepsinogengruppe $I$, Pg III = Pepsinogengruppe II. 


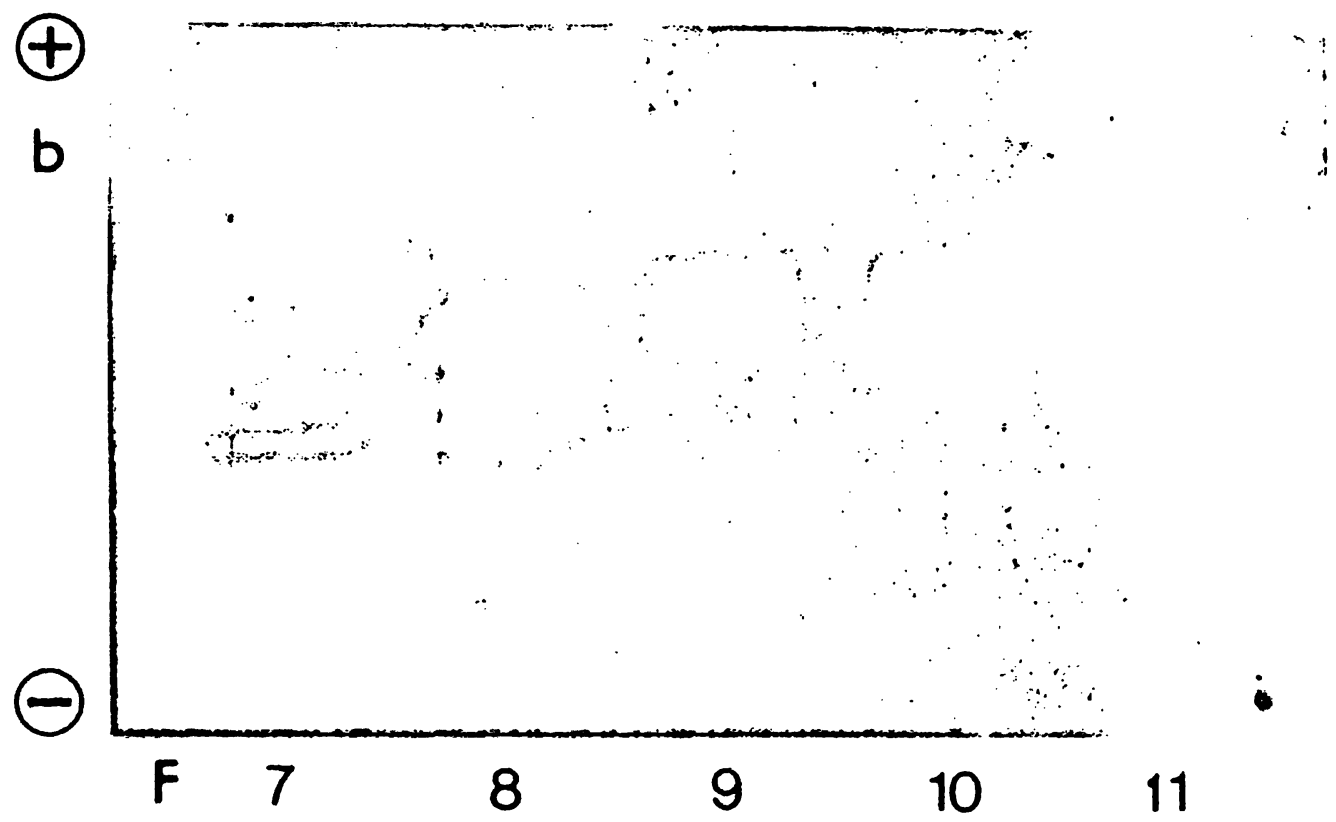

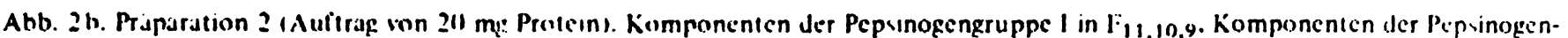

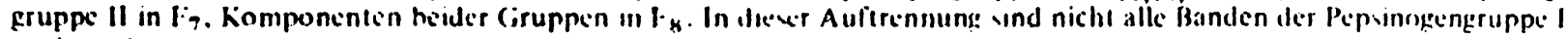
nachicentiar.

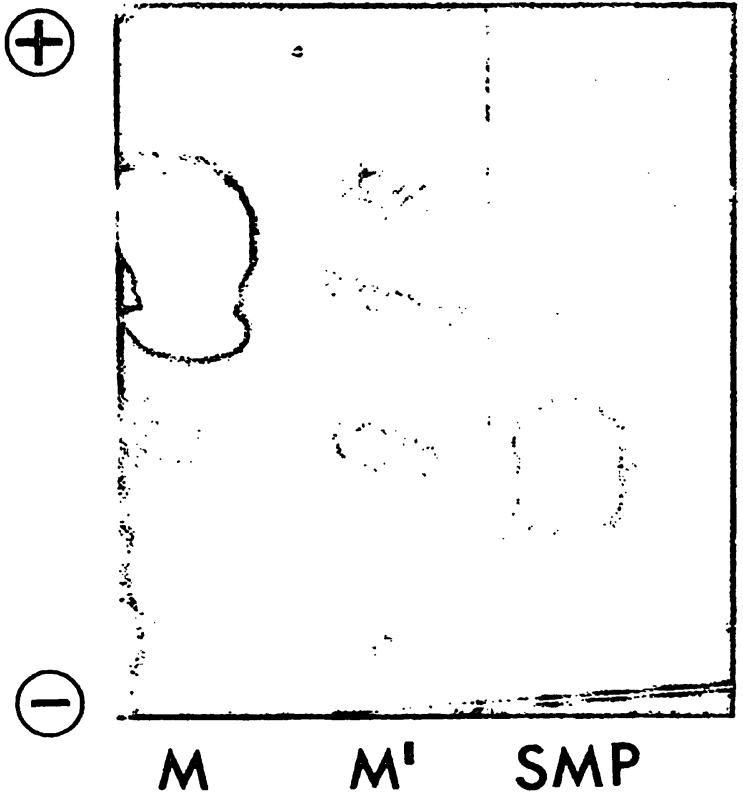

Abb. 3. Agargelenzymelektrophorese isolierter Fraktionen aus angesäuertem und neutralisiertem Magenextrakt. $\mathbf{M}=$ Ausgangsmaterial. $\mathbf{M}^{\prime}=$ Magenexirakt nach Ansäuerung und Neutralisierung. SMP = Reine Fraktion der langsam wandernden Magenschleimhautprotease.

\section{Herstellung von Antiseren}

Die zur Immunisierung verwendeten Proteinfraktionen der Pepsinogengruppe I bzw. II werden in Abbildung 4 dargestellt. Die zur Immunisierung verwendete langsame Protease wird in Abbildung 3 gezeigt. Die Immunisierung von einem Schaf und 3 Kaninchen mit den isolierten

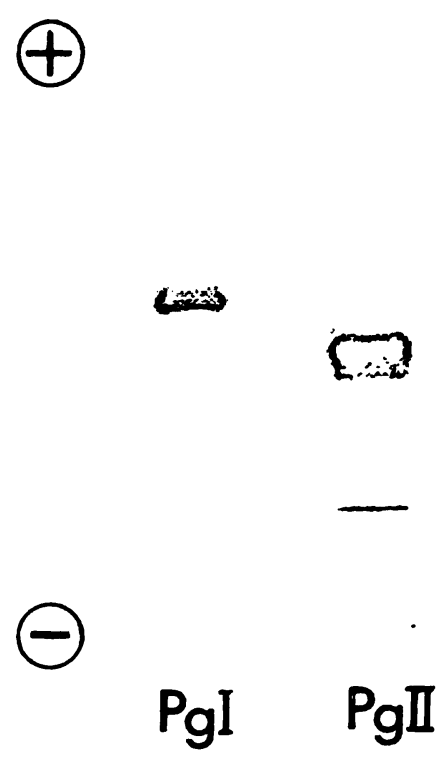

Abb. 4. Elektrophoretische Darstellung der zur Immunisierung verwendeten Komponenten der Pepsinogengruppe I (Pg I) und der Pepsinogengruppe II (Pg II).

Komponenten der Pepsinogengruppe I führte zu Antiseren, die in der Geldiffusion und in der Immunelektrophorese jeweils nur eine Präzipitationslinie gegen Magentotalextrakte ergaben (Abb. 5a). Antikörper gegen Serumproteine waren nicht nachweisbar. Die Immunisierung von einem Schaf und vier Kaninchen mit den isolierten Komponenten der Pepsinogengruppe II ergab Antiseren, 
die in der Geldiffusion und in der Immunoelektrophorese die typische Präzipitationslinie gegen die Pepsinogengruppe II zeigten (Abb. 5a). Bei zwei Kaninchen waren noch in Spuren Antikörper gegen Serumalbumin vorhanden. Zwei Kaninchen wurden mit der langsam wandernden Protease immunisiert. Auch hier zeigten die Antikörper spezifísche Präzipitationslinien gegen die langsam wandernde Protease in Magentotalextrakten. Wie in früheren Untersuchungen war diese Präzipitationslinie sehr schwach ausgeprägt (Abb. 5a). In der zweidimensionalen Immunelektrophorese konnten wir unter der Verwendung der beiden monospezifischen Antiseren die immunchemische Heterogenität der Pepsinogengruppe I und II demonstrieren (Abb. 5b).

\section{Diskussion}

Aufgrund der bisherigen Untersuchungen hat sich gezeigt, daß die menschlichen Pepsinogene zwei unterschiedlichen Systemen zugeordnet werden können. Hierfür sprechen immunchemische Unterschiede $(4,20,21,22)$, unterschiedliche elektrophoretische Verteilungsmuster (8), unterschiedliche topographische Verteilung der die beiden Gruppen sezernierenden Zellen der Magenschleimhaut $(23,24)$ sowie Unterschiede in der renalen Sekretionsfähigkeit für Pepsinogen I und II (20). Die beiden
Pepsinogensysteme sind mikroheterogen, d. h. sie bestehen aus chromatographisch und elektrophoretisch unterscheidbaren Komponenten (4, 8, 20, 25, 26, 27, 28, 29), ähnlich der Isoenzyme. Die verschiedenen Komponenten lassen sich am besten in der Enzymelektrophorese darstellen.

Ein drittes, immunchemisch definierbares und elektrophoretisch langsam wanderndes System von Proteasen der menschlichen Magenschleimhaut wurde in dem elektrophoretischen Bereich von Albumin nachgewiesen $(3,4)$. Es unterscheidet sich von den beiden Pepsinogengruppen durch seine geringe Konzentration in den Magenschleimhautextrakten, durch seine deutliche elektrophoretische Absetzung von der Pepsinogengruppe II und durch seine Stabilität in neutralem und alkalischem Milieu nach erfolgter Säureaktivierung $(4,8)$.

Mit der präparativen Polyacrylamid-Gel-Elektrophorese konnten wir in einer Gelkonzentration von $15 \%$ die Proteasen von zahlreichen in den Magenschleimhautextrakten vorhandenen Organ- und Plasmaproteinen abtrennen. Dies wurde durch die relativ große elektrophoretische Mobilität der Pepsinogengruppe I und Pepsinogengruppe II sowie durch deren relativ geringes Molekulargewicht ermöglicht. Die Ausbeute pro Auftrennung war mit 0,5-0,9 mg sehr gering, reichte jedoch zur Herstellung von hochspezifischen Immunseren aus. Allerdings

\section{$a \oplus$}

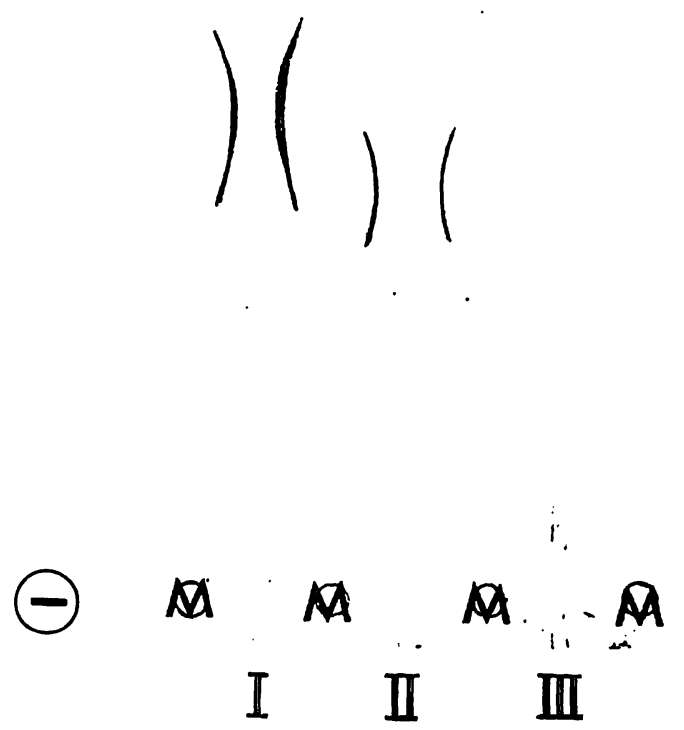

Abb. 5. Nachweis monospezifischer Antiseren gegen die Pepsinogengruppen I und II.

Abb. 5a. Immunelektrophoretische Auftrennung von Magentotalextrakt (M).

I = Antikörper gegen Pepsinogengruppe I

II = Antikörper gegen Pepsinogengruppe II

III = Antikörper gegen langsam wandernde Magenschleimhautproteasen.

b

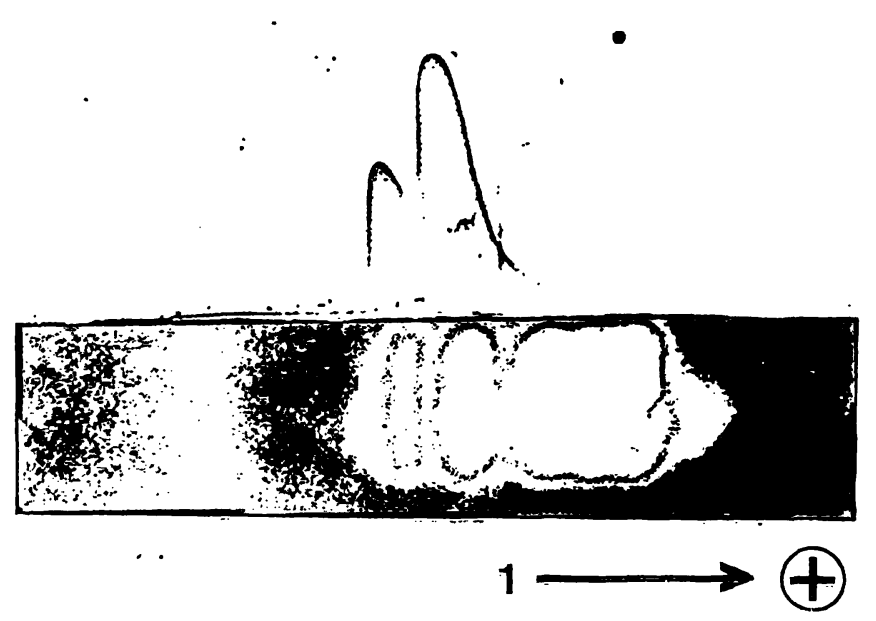

Abb. 5 b. Zweidimensionale Immunelektrophorese.

Unten: Simultane Darstellung der Pepsinogengruppen I und II, enthalien in Magenextrakt mit Hilfe der Enzymclektrophorese. Es sind nicht alle Komponenten der Pepsinogengruppe I vorhanden.

Oben: Immunelektrophoretische Auftrennung. Im Gel ein Gemisch von monospezifischen Antiseren, gerichtet gegen die Pepsinogengruppen I und II. 
ist hierbei zu berücksichtigen, daß der Arbeitsau fwand im Vergleich zu der relativen Reinheit der einzelnen Pepsinogenkomponenten gering war.

In den einzelnen Unterfraktionen nach präparativer Polyacrylamid-Gel-Elektrophorese konnten alle in dem Ausgangsmaterial nachweisbaren Komponenten der Pepsinogengruppen I und II wiedergefunden werden. In den einzelnen Polyacrylamid-Gel-Elektrophorese-Fraktionen war die Auftrennung in der Enzymelektrophorese sogar wesentlich besser als im Ausgangsmaterial. So zeigte sich deutlich, daß die Pepsinogengruppen I und II in der Enzymelektrophorese mindestens aus insgesamt acht Komponenten bestehen. Die ersten fünf anodenwärts gelegenen Komponenten (Banden 1-5) entsprechen der von Samloff beschriebenen Pepsinogengruppe I. In der zweidimensionalen Elektrophorese konnten wir zeigen, daß die erste rasch anodisch wandernde Bande ebenfalls zu dem immunchemisch definierbaren System der Pepsinogengruppe I geliört.

In der elektrophoretisch definierbaren Pepsinogengruppe Il fanden wir eine dritte Komponente. Aus laufenden Untersuchungen geht allerdings hervor, daß die Zahl der in der Pepsinogengruppe II vorhandenen Komponenten noch größer sein dürfte. Trotz der relativ scharf trennenden Polyacrylamid-Gel-Elektrophorese war es uns nicht möglich gewesen, alle Komponenten der Pepsinogengruppe II von jenen der Pepsinogengruppe I zu trennen. Wir nehmen an, daß die Komponenten mittlerer elektrophoretischer Mobilität, d. h. die Banden 4 und 5 (Pepsinogengruppe I) und 6 und 7 (Pepsinogengruppe II) nahezu identische elektrische Ladungen besitzen und derart zu einer Überlappung führen. Diese Beobachtung erklärt auch die Erfolglosigkeit bisheriger Versuche, beide immunchemisch differente Systeme mit all e n Komponenten aus Magenextrakten zu isolieren. Die von uns isolierten Teilkomponenten der Pepsinogengruppe I und II erwiesen sich jedoch aufgrund ihrer immunchemischen Identität als ausreichend immunogen, um hochspezifische Antiseren gegen die Gesamtheit der Pepsinogengruppe I bzw. Pepsinogengruppe II zu gewinnen. Dies zeigte sich deutlich in der zweidimensionalen Immunelektrophorese.

Durch die Ansäuerung mit nachfolgender Alkalisierung der Magenextrakte wurde die Pepsinogengruppe I bzw. ihre aktive Komponente Pepsin I inaktiviert. Dagegen wurde wider Erwarten die Pepsinogengruppe II bzw. Pepsin II nur teilweise inaktiviert, denn in einer elektrophoretischen Zone waren noch proteolytische Aktivitäten vorhanden. In der Geldiffusion waren noch immunoreaktive Bestandteile der Pepsinogengruppe II in der Geldiffusion nachzuweisen. Aufgrund dieser Beobachtung nehmen wir an, dạß zwischen der Pepsinogengruppe I und der Pepsinogengruppe II auch Unter- schicde hinsichtlich der Alkaliinaktivierung bestehen. Die quantitativ-immunolo ische Bestimmung der $\mathrm{Bc}$ standteile der Pepsinogengruppe II nach Inaktivierung ergab wesentlich höhere Werte als in dem Ausgangsmaterial. Desgleichen waren auch in den Fraktionen der präparativen Polyacrylamid-Gel-Elcktrophorese wesentlich höhere immunologisch faßbare Konzentrationen als enzymatische Aktivitäten nachzuweisen. Dieses paradoxe Phänomen läßt sich am besten durch das Auftreten von immunchemisch aktiven Spaltprodukten oder aber durch unterschiedliche Diffusionseigenschaften der aktivierten Pepsinogengruppe II erklären.

Die langsam wandernde Magenprotease wandert zusammen mit Albumin und weiteren Organ-bzw. Serumproteinen in einem umschriebenen elektrophoretischen Bereich, so daß eine Isolierung aus nativen Organextrakten mit den klassischen Techniken nur schwer vorstellbar ist. Da die langsam wandernde Protease jedoch gegenüber der proteolytischen Wirkung der Pepsinogengruppe I bzw. Il resistent und dazu noch alkalisch stabil ist, haben wir die Magenextrakte durch Ansäuerung einer Eigenverdauung und die Pepsine durch Alkalisierung einer Denaturierung unterzogen. Bereits nach einer Auftrennung mit präparativer Polyacrylamid-Gel-Elektrophorese war es möglich, die langsam wandernde Protease von den noch immunreaktiven Bestand teilen der Pepsinogengruppe II zu trennen. Die nach einer zweiten Auftrennung isolierte langsam wandernde Protease ergab in der Enzymelektrophorese zwei bis drei Komponenten mit proteolytischer Aktivität. Diese elektrophoretische Heterogenität der langsam wandernden Protease entspricht den Beobachtungen von Mangla et al. (30), der kürzlich diese Proteasen als Cathepsin identifizierte.

In der vorliegenden Untersuchung haben wir mit der in ihrer Trennschärfe unübertroffenen Methode der Polyacrylamid-Gel-Elektrophorese gezeigt, daß es nicht möglich ist, mit den klassischen Trennverfahren die menschlichen Pepsinogengruppen I und II mit allen ihren Komponenten voneinander zu trennen. Dennoch war es möglich, durch die Isolierung von Teilkomponenten gruppenspezifische Immunseren zu gewinnen.

\section{Danksagung}

Wir danken Herrn Prof. Dr. R. C. Allen und Herrn Prof. Dr. $B$. Urbaschek für ihre Unterstützung anläßlich des Aufbaus der Polyacrylamid-Gel-Elektrophoresen, sowie Frau $\boldsymbol{M}$. Windisch für ihre wertvolle technische Assistenz. Wir danken Herrn Mechanikermeister $E$. Meier, Medizinische Universitätsklinik Heidelberg, für seine Mithilfe bei dem Aufbau der präparativen Einrichtungen. Die Magenpräparate wurden licbenswürdigerweise von Herrn Chefarzt Dr. G. Kolig, St. Josefskrankenhaus Heidelberg, zur Verfügung gestellt. 


\section{Literatur}

1. Uriel, J. (1960), Nature (London) 188, 853-854.

2. Rapp, W., Aronson, S. B. \& Burtin, P. (1963), Verh. Deutsch. Ges. Inn. Med. 69, 153-155.

3. Rapp, W., Aronson, S. B., Burtin, P. \& Grabar, P. (1964), J. Immunol. 92, 579-595.

4. Kushner, I., Rapp, W. \& Burtin, P. (1964), J. Clin. Invest. 43, 1983-1993.

5. Hirsch-Marie, H., Conte, M. \& Burtin, P. (1965), Rev. Franc. Etudes Clin. Biol. 10, 924-934.

6. Hirsch-Marie, H. (1966), Thèse Doct., Fac. Science, Paris.

7. Samloff, M., Barnett, E. V.\& Turner, M. D. (1967), Gastroenterology $52,165-175$.

8. Samloff, I. M. (1969), Gastroenterology 57, 659-669.

9. Whitecross, D. P., Armstrong, C., Clarke, A. D. \& Piper, D. W. (1973), Gut 14, 850-855.

10. Mangla, J. C., Guarasci, G. \& Turner, M. D. (1973), Digest. Diseases 18, 857-864.

11. Zöller, M., Matzku, S. \& Rapp, W. (1975), Biochim. Biophys. Acta $427,708-718$.

12. Rapp, W. \& Lehmann, H. E. (1972), Europ. J. Clin'. Invest. 2, 243-249.

13. Mancini, G., Carbonara, A. O. \& Heremans, J. F. (1965), Immunochemistry 2, 235-243.

14. Rapp, W. (1967), Clin. Chim. Acta 15, 177-179.

15. Allen, R. C., Moore, D. J. \& Dilworth, R. H. (1969), J. Histochem. Cytochem. 17, 189-194.
16. Maurer, H. R. (1971), Disc-Elektrophorese, Walter de Gruytcr, Berlin-New York, p. 50.

17. Laurell, C. B. (1965), Anal. Biochem. 10, 358-361.

18. Lowry, O. H., Rosebrough, N. J., Farr, A. L. \& Randall, R. J. (1951), J. Biol. Chem. 193, 265-275.

19. Rick, W. \& Fritsch, W. P. (1974) in Methoden der enzymatischen Analyse Bd. 1, 1087-1097. (H. U. Bergmeyer, ed.) Verlag Chemie, Weinheim/Bergstr.

20. Samloff, M. \& Townes, Ph. L. (1970), Gastroenterology 58 , $462-469$.

21. Samloff, I. M. (1971), Gastroentcrology 60, 586-604.

22. Samloff, I. M. (1971), J. Immunol. 106, 962-968.

23. Samloff, I. M. (1971), Gastroentcrology 61, 185-188.

24. Samloff, I. M. \& Liebman, W. M. (1973), Gastroentcrology $65,36-42$.

25. Seijeffers, M. J., Segal, H. L. \& Miller, L. L. (1963), Am. J. Physiol. 205, 1099-1105.

26. Hanley, W. B., Boyer, S. H. \& Naughton, M. A. (1966), Nature 209, 996-1002.

27. Turner, M. D., Mangla, J. C., Samloff, I. M., Miller, L. L. \& Segal, H. L. (1970), Biochem. J. 116, 397-404.

28. Bowen, P. (1974), Am. J. Hum. Gen. 26, 16 A.

29. Turner, M. D. \& Miller, L. L. (1965), Am. J. Gastroenterol. 44, 431-439.

30. Mangla, J. C., Guarasci, G. \& Turner, M. D. (1974), Biochem. Med. 10, 83-96.
Prof. Dr. W. Rapp

Bergheimer Str. 58 D-6900 Heidelberg 